\title{
Engagement, workers' strategies, therapeutic relationships, coercion and outcomes in community mental health services in Northern Ireland Gavin Davidson
}

\author{
Address: School of Sociology, Social Policy and Social Work, Queen's University Belfast, College Park, Belfast, BT7 1NN, UK \\ from WPA Thematic Conference. Coercive Treatment in Psychiatry: A Comprehensive Review \\ Dresden, Germany. 6-8 June 2007 \\ Published: 19 December 2007 \\ BMC Psychiatry 2007, 7(SuppI I):S53 doi:I0.1 I86/I47|-244X-7-SI-S53
}

This abstract is available from: http://www.biomedcentral.com/I47I-244X/7/SI/S53

(c) 2007 Davidson; licensee BioMed Central Ltd.

\section{Background}

To explore possible associations between the level of engagement, the strategies workers use to encourage treatment compliance, the therapeutic relationship, perceived coercion and outcomes in a cohort of 'difficult-to-engage' clients receiving either Community Mental Health Team (CMHT) support or Assertive Community Treatment (ACT) support in Northern Ireland. This study also aimed to inform the current review of mental health services in Northern Ireland which is considering service organization and the possible introduction of community treatment orders.

\section{Methods}

A quasi-experiment design was used as it was not possible to randomly allocate to the CMHT and ACT groups. 76 people met the inclusion criteria and received support over 18 months. Data was collected at 0,9 and 18 months. 60/76 consented to involvement at baseline and it was possible to collect data on 55/60 at 18 months. Inpatient data was collected for all 76 . The main scales used were the Service Engagement Scale, the Therapeutic Limit Setting scale, the Working Alliance Inventory and the Perceived Coercion Scale.

\section{Results}

In accordance with most US studies of assertive community treatment but in contrast to recent UK studies, this study found it to be effective at reducing the need for both voluntary and involuntary inpatient hospital use. In considering the therapeutic relationship only $24 / 55$ clients had the same key-worker after 18 months.

\section{Conclusion}

Assertive community treatment should be considered as an effective means of engaging clients, building trusting relationships and promoting stability and may obviate the need to introduce more coercive measures such as community treatment orders. 